\title{
Avaliação do aumento da reatividade da cinza volante através do método sonoquímico
}

\author{
Evaluation of the increase fly ash reactivity through \\ the sonochemistry method
}

\author{
Alini Luísa Diehl Camacho ${ }^{1}$, David Lamberto Diehl Camacho ${ }^{2}$, \\ Maurício Mancio ${ }^{2}$
}

\author{
${ }^{1}$ Laboratório de Caracterização e Valorização de Materiais - LCVMat - UNISINOS CEP: 93022-750, São Leopoldo, RS, \\ Brasil. \\ e-mail: aldiehl@unisinos.br \\ ${ }^{2}$ Programa de Pós-Graduação em Engenharia Civil - PPGEC- UNISINOS CEP: 93022-750, São Leopoldo, RS, Brasil. \\ e-mail:davidlc@unisinos.br; mancio@unisinos.br
}

\begin{abstract}
RESUMO
Este trabalho teve como objetivo avaliar o aumento da reatividade da cinza volante (CV) através do método sonoquímico combinado ao processo de sifonação da CV, a fim de separar materiais constituintes por fases, sendo elas amorfas e cristalinas. Em seguida, foram realizados tratamentos sonoquímicos em dois tipos de equipamentos como forma de comparação. Os materiais tratados foram caracterizados pelas técnicas de tamanho de partícula, massa específica, área superficial, composição química e mineralógica, e por fim, moldados conforme a norma NBR 5752:2014. O método Chapelle modificado também foi aplicado para verificar o consumo de hidróxido de cálcio. Como estudo complementar, foram realizadas análises microestruturais da $\mathrm{CV}$ antes e depois da aplicação do método sonoquímico utilizando a microscopia eletrônica de varredura. Os resultados de resistência à compressão comprovaram que o método utilizado contribuiu para um aumento de 84 para $112,9 \%$ do índice de pozolanicidade do material associado à aplicação do ultrassom. As amostras CV NAT, CV ULT SP e CV ULT UNI foram capazes de fixar maior quantidade de cal do que a CV SIF. Entretanto, o índice de atividade pozolânica, por meio da hierarquização indireta indica um comportamento favorável com a possibilidade, inclusive, de desclassificar a CV como material pozolânico, caso ela não passe por um processo de beneficiamento. Nas imagens microestruturais verificou-se uma redução no tamanho das partículas, causado, provavelmente pela quebra das pleosferas da $\mathrm{CV}$.
\end{abstract}

Palavras-chave: Cinza volante, ultrassom, ativação sonoquímica, índice de pozolanicidade.

\section{ABSTRACT}

The objective of this work was to evaluate the increase of fly ash reactivity $(\mathrm{CV})$ through the sonochemistry method combined with the CV siphoning process, in order to separate constituent materials by phases, being amorphous and crystalline. After that, sonochemistry treatments were performed in two types of equipment as a way of comparison. The treated materials were characterized by the techniques of particle size, specific mass, surface area, chemical and mineralogical composition, and, finally, molded according to the norm NBR 5752: 2014. The modified Chapelle method was also applied to verify the consumption of calcium hydroxide. As a complementary study, microstructural analysis of CV was performed before and after the application of the sonochemistry method using scanning electron microscopy. The results of compressive strength proved that the method used contributed to an increase from 84 to $112.9 \%$ of the pozzolanicity index of the material associated with the application of ultrasound. The samples CV NAT, CV ULT SP and CV ULT UNI were able to fix higher amount of lime than CV SIF. However, the index of pozzolanic activity, through indirect hierarchization indicates a favorable behavior with the possibility, even, of declassifying the $\mathrm{CV}$ as pozzolanic material, if it is not submitted to a beneficiation process.. In microstructural images there was a reduction in particle size, probably caused by the breakdown of the CV pleospheres.

Keywords: Fly ash, ultrasound, activation sonochemistry, pozzolanicity activity. 


\section{INTRODUÇÃO}

LEVANDOWSKI e KALKREUTH [1] indicam que o Brasil possui, especificamente no Estado do Rio Grande do Sul, sete usinas termelétricas que produzem cerca de 3 milhões de t/cinzas por ano, as quais 65 a $85 \%$ correspondem à fração leve e de 15 a $35 \%$ à fração pesada. Como é previsto um aumento de mais de $100 \%$ no consumo de carvão para fins termelétricos no Brasil, a geração deste resíduo deve chegar de 3,5 a 4 milhões de t/ano [2]. A produção anual a nível mundial de cinzas de carvão é estimada em torno de 600 milhões de toneladas e 500 milhões de toneladas para CV que corresponde de 75 a $80 \%$ do total de cinzas geradas [3].

Em contrapartida, as adições minerais ativas e inertes na produção mundial do cimento Portland representam cerca de $86 \%$ em sua composição (cinza volante, argila calcinada e escória granulada de alto forno, sendo o fíller calcário utilizado como adição inerte), fato este que em 2013 chegou a 70,2 milhões de toneladas produzidas [4]. O grande volume destas adições à composição do cimento Portland evidencia sua importância frente à caracterização, seleção e cinética de reações destes materiais nos efeitos da hidratação do cimento, bem como no comportamento da sua reatividade, que pode ser tanto física quanto química, em especial, a CV que é adicionada em torno de 50\% no cimento Portland CP IV. [5]

As interações físicas causadas com os produtos de hidratação do cimento modificam a microestrutura do material da zona de transição entre a pasta de cimento e o agregado, reduzindo ou eliminando o acúmulo de água livre que fica retida sob os agregados. Podem ser gerados vários efeitos através destas adições como: efeito microfíller, produto do aumento da densidade da mistura pelo preenchimento de espaços vazios pelas partículas das adições, cujo diâmetro médio deve ser semelhante ou menor que o diâmetro médio das partículas do cimento, refinamento da estrutura dos poros, ocasionado pelas partículas das adições que podem agir como pontos de nucleação para os produtos de hidratação. Por outro lado, o comportamento químico é decorrente da capacidade de reação da adição mineral com o hidróxido de cálcio hidratado $\left[\mathrm{Ca}(\mathrm{OH})_{2}\right]$, gerado durante a hidratação do cimento para formar silicato de cálcio hidratado adicional (C-S-H) [5].

A utilização do ultrassom em nanomateriais tem sido estudada na dispersão de materiais em líquidos para a quebra de aglomerados. Normalmente o efeito da cavitação que ocorre durante as ondas ultrassônicas leva a partículas menores e consegue-se uma uniformidade no tamanho das partículas. Contudo, a cavitação ultrassônica melhora a transferência de material na superfície das partículas e pode ser utilizada para aumentar a área superficial [6,7]. A aplicação sonoquímica permite bons rendimentos, elevada cristalinidade dos produtos e proporciona uma redução considerável do tempo de reação de dias para minutos [7,8].

Quando dispersas em um meio líquido, as partículas ficam rodeadas por uma camada limite de moléculas atraídas para a superfície da partícula que precisa ser removida. O fenômeno sonoquímico causa um estresse superando as forças de atração e transportando as moléculas para a superfície da partícula [7]. Além disso, alterações na morfologia superficial das partículas podem ser induzidas, incluindo a redução do tamanho delas. Muitas vezes, o processo sonoquímico aumenta, de maneira efetiva, a reatividade dos materiais e a formação de fases aluminosas ocorre em temperaturas, no mínimo, $100^{\circ} \mathrm{C}$ menores do que as produzidas convencionalmente sem a utilização do processo sonoquímico [9].

SUSLICK [6] e GEDANKEN [7] acreditam que a explicação do fenômeno de cavitação é de que a cinética rápida não permite o crescimento dos núcleos. Em cada bolha gerada no colapso, são formados alguns centros de nucleação cujo crescimento é limitado. A implosão das bolhas geradas na cavitação resulta em micro turbulências e micro jatos de até $1000 \mathrm{~km} / \mathrm{h}$ de partículas grandes que são sujeitas a erosão superficial ou redução de tamanho de partícula, isto leva à aceleração da difusão (causando aumento na temperatura), processos de transferência de massa e as reações de fase sólida [6].

As adições minerais nos cimentos, bem como a utilização de materiais pozolânicos, destacado aqui a $\mathrm{CV}$, são caracterizadas por serem responsáveis pela capacidade de reagir com o hidróxido de cálcio liberado na hidratação do cimento, em temperatura ambiente e na presença de água, formando compostos com propriedades hidráulicas [10]. Métodos indiretos de quantificar o potencial reativo destas adições minerais são comumente utilizados por meio do índice de atividade pozolânica (IAP). Este índice de atividade pozolânica é a relação percentual entre a resistência média de um material com adição pozolânica e o mesmo material sem sua adição (referência). A norma NBR 12653 [11] estabelece um valor mínimo de 90\% para que o material seja considerado pozolânico. O método direto comumente utilizado, denominado ensaio de Chapelle modificado [12] determina a capacidade da adição pozolânica em fixar cal para a formação de compostos hidratados.

A cinza volante $(\mathrm{CV})$ é um resíduo industrial gerado na queima do carvão mineral em usinas termelétricas, classificada como adição mineral com baixo teor de cálcio. O pó gerado corresponde de 15-30\% de partículas maiores de $45 \mu \mathrm{m}$ sendo a maioria destas partículas esféricas e sólidas com diâmetro menor que 20 $\mu \mathrm{m}$. Em função de suas características microestruturais, a CV apresenta pleosferas (parte externa das esferas 
consideradas partículas ocas) e, em seu interior, partículas amorfas (cenosferas) localizadas internamente [10].

Desta forma, este artigo tem como objetivo avaliar o comportamento do material CV quando submetido ao processo sonoquímico. Para verificar se ocorre aumento da reatividade física e química, os métodos de avaliação de índice de atividade pozolânica $[12,13,14]$ foram realizados, bem como a caracterização física, química, mineralógica e microestrutural do material.

\section{MATERIAIS E MÉTODOS}

Para o desenvolvimento deste estudo a amostra CV utilizada foi proveniente da usina termelétrica de Candiota localizada no estado do Rio Grande do Sul, Brasil.

O método sonoquímico aplicado ao material foi realizado pelo equipamento Ultrasonic Processor UIP1000hd. Este ultrassom foi escolhido em virtude de sua aplicação específica na área da sonoquímica e também pela potência de 1000 Watts. Além deste equipamento, como forma de comparação na aplicação do método sonoquímico, também foi utilizado um ultrassom de bancada, marca UNIQUE, modelo USC2850, capacidade de 9,5 litros, $25 \mathrm{kHz}$, potência de 100 Watts. Neste ultrassom, durante o ensaio foi acoplado um agitador mecânico, potência 25 Watts que ajudou na dispersão do material.

\subsection{Metodologia}

A metodologia utilizada é ilustrada conforme Figura 1. Os métodos de caraterização dos materiais e avaliação da reatividade pozolânica após o tratamento sonoquímico estão descritos a seguir.

Preparou-se uma suspensão aquosa, numa relação água/sólido na proporção de 30:1 [9]. Essa suspensão foi submetida ao processo ultrassônico por 1 hora com agitação mecânica constante no equipamento Ultrasonic Processor UIP1000hd. Como os equipamentos possuem potências diferentes, no equipamento Unique, a amostra permaneceu por 8 horas, de forma a manter uma mesma quantidade de trabalho em ambos equipamentos. Após o tratamento, o material foi seco em estufa a $105^{\circ} \mathrm{C}$ por 24 horas e realizou-se a caracterização. A CV bruta foi denominada como CV NAT, a CV tratada no ultrassom de marca Unique foi denominada CV ULT UNI, a CV tratada no ultrassom UIP1000hd foi denominada CV ULT SP, a CV que foi apenas beneficiada pela sifonação foi denominada como CV SIF e a CV que sofreu o processo de sifonação com aplicação do ultrassom Unique foi denominada de CV SIF+ULT UNI.

O processo de sifonação ocorreu em 8 etapas. Etapa 1: preparo da solução de hexametafosfato de sódio $\left(\mathrm{NaPO}_{3}\right)_{6}$; etapa 2: mistura e agitação manual; etapa 3: período de decantação para separação da fração maior e mais densa da fração mais fina e menos densa (predominantemente vítrea); etapa 4: sifonação da solução com partículas vítreas em suspensão; etapa 5: centrifugação ou novo período de decantação para separação entre a água e as partículas vítreas; etapa 6: sifonação da água; etapa 7: secagem da fração vítrea em estufa a $105^{\circ} \mathrm{C}$ até massa constante e; etapa 8: remoção da fase vítrea denominada CV SIF.

O material foi previamente caracterizado antes e depois da aplicação do método sonoquímico através das técnicas de caracterização:

Caracterização física: tamanho de partículas, massa específica e área superficial.

Caracterização química e mineralógica: fluorescência de raios X (FRX) e difração de raios X (DRX).

Caracterização microestrutural por microscopia eletrônica de varredura.

Avaliação do índice de atividade pozolânica. 


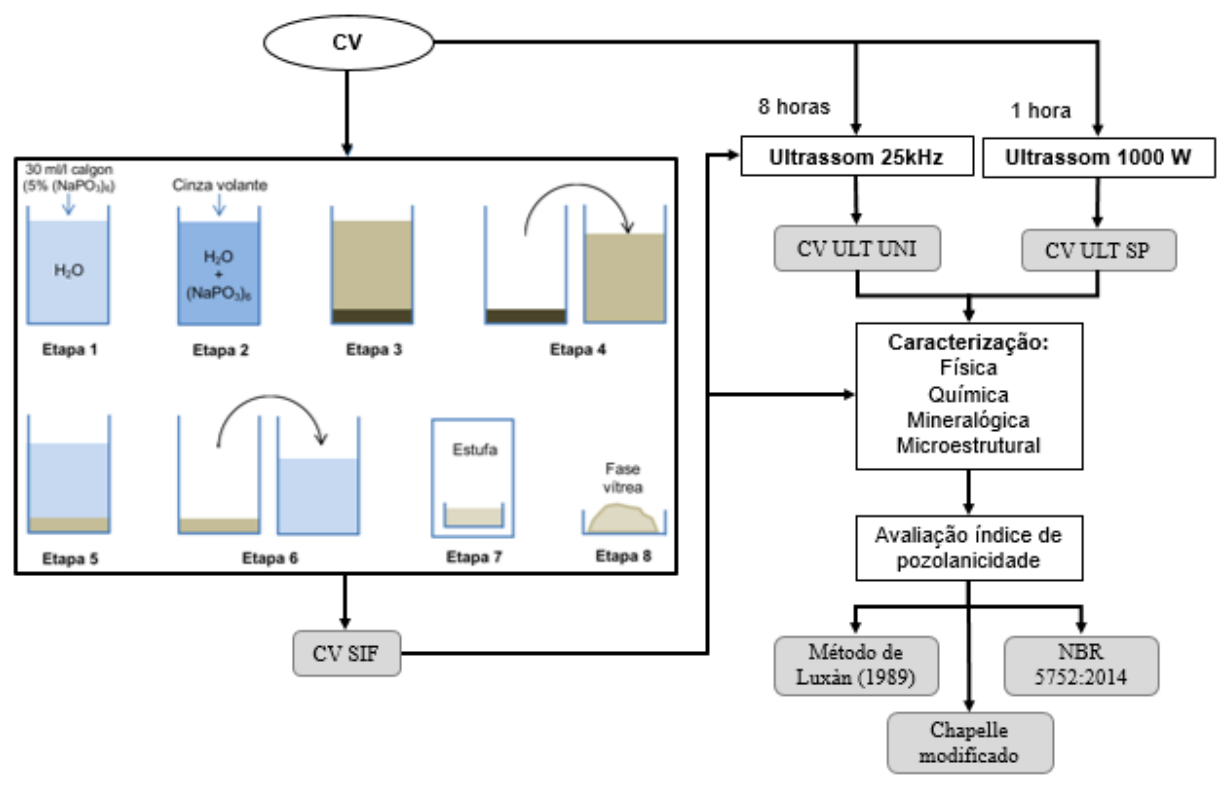

Figura 1: Fluxograma do programa experimental.

\subsection{Caracterização física: tamanho de partícula, massa específica e área superficial}

O diâmetro médio, bem como a distribuição do tamanho das partículas dos materiais foram determinados por granulometria a laser, método via úmido, equipamento de marca MICROTRAC, modelo S3500 [15].

A massa específica real dos materiais foi determinada via picnometria a gás hélio, pelo equipamento de marca MICROMERITICS, modelo ACCUPYC 1340. A área superficial foi determinada pelo método BET (Branauer, Emmett e Teller) por equipamento marca MICROMERITICS, modelo Tristar Plus II.

\subsection{Caracterização química e mineralógica}

A composição química quantitativa foi determinada por espectrometria de Fluorescência de Raios X utilizando curva de calibração com padrões de referência para Fly Ash da Brammer, sendo eles CRM SABS 109, CRM NCS FC82015a e CRM NCS FC82017a. O equipamento utilizado foi um Espectrômetro de Fluorescência de Raios X por energia dispersiva, marca Shimadzu, modelo EDX 720 HS. A fração orgânica L.O.I. (Loss on ignition) foi realizada de acordo com a norma CEMP $n^{\circ} 120$ [16].

Para identificação da composição mineralógica dos materiais foi utilizada a técnica de Difração de Raios X, pelo equipamento marca Siemens, modelo 5000, sendo o software para interpretação das fases cristalinas utilizado o X'Pert HighScore da Philips. O método de referência de proporção e intensidade (RIR) foi empregado para determinar o conteúdo das fases cristalinas semi-quantitativas com respectivo banco de dados.

\subsection{Caracterização microestrutural}

A técnica de Microscopia Eletrônica de Varredura (MEV) com EDS para determinação pontual de composição química foi realizada num equipamento de marca ZEISS, modelo EVO MA15. As imagens foram tratadas com uso do software FIJI ImageJ. As amostras foram pulverizadas, fixadas no stub em fita de carbono e metalizadas com ouro.

\subsection{Avaliação do índice de atividade pozolânica}

A atividade pozolânica foi medida de acordo com método indireto Luxàn et al. [13] e através da determinação da resistência à compressão simples [17].

Para verificar se houve aumento na reatividade química dos materiais realizou-se a técnica direta através da determinação do teor de hidróxido de cálcio fixado, conhecido como Chapelle modificado. O método é aplicado a materiais silicosos e sílico-aluminosos, cuja fase amorfa tenha a capacidade de fixar hidróxido de cálcio [12].

O cálculo é realizado considerando $2 \mathrm{~g}$ de $\mathrm{CaO}$ e $1 \mathrm{~g}$ do material pozolânico, conforme equação 1 : 


$$
\frac{m g \mathrm{CaO}}{g} \text { de material }=\frac{28 *\left(V_{3}-V_{2}\right) * F_{C}}{m_{2}} * 1,32
$$

Onde:

$m_{2}$ : gramas do material pozolânico;

$V_{2}$ : é o volume em $\mathrm{mL}$ de $\mathrm{HCl} 0,1 \mathrm{M}$ consumido durante a titulação com a amostra;

$V_{3}$ : é o volume em $\mathrm{mL}$ de $\mathrm{HCl} 0,1 \mathrm{M}$ consumido durante a titulação no ensaio em branco;

$f_{C}$ : fator de correção do $\mathrm{HCl}$ para uma concentração de $0,1 \mathrm{M}$;

1,32: é a relação molecular $\mathrm{Ca}(\mathrm{OH})_{2} / \mathrm{CaO}$.

\section{RESULTADOS E DISCUSSÕES}

\subsection{Caracterização física}

Os resultados de tamanho de partículas por granulometria a laser estão ilustrados na Tabela 1. Verificou-se que houve uma redução no tamanho das partículas da amostra CV NAT em comparação com a amostra CV ULT UNI, uma variação de aproximadamente $43 \%$ em relação ao diâmetro médio, demonstrando a eficiência do método sonoquímico na diminuição do tamanho das partículas. A redução do tamanho das partículas também foi verificada na amostra CV ULT SP e na CV SIF. No tratamento combinado CV SIF+ULT UNI verificou-se que o $\mathrm{D}_{10}$ aumentou de tamanho em relação a CV NAT. Este aumento pode estar associado ao fato de uma possível aglomeração das partículas na faixa inicial de sua distribuição $\left(\mathrm{D}_{10}\right)$.

Tabela 1: Resultados de tamanho de partículas por diâmetro equivalente.

\begin{tabular}{l|l|l|l|l|l}
\hline DIMENSÕES & \multicolumn{1}{|c|}{ CV NAT } & CV ULT UNI & CV ULT SP & \multicolumn{1}{c}{ CV SIF } & CV SIF + ULT UNI \\
\hline Diâmetro a 10\% & $5,84 \mu \mathrm{m}$ & $4,48 \mu \mathrm{m}$ & $3,54 \mu \mathrm{m}$ & $5,31 \mu \mathrm{m}$ & $9,97 \mu \mathrm{m}$ \\
\hline Diâmetro a $50 \%$ & $43,30 \mu \mathrm{m}$ & $28,70 \mu \mathrm{m}$ & $16,72 \mu \mathrm{m}$ & $17,48 \mu \mathrm{m}$ & $35,03 \mu \mathrm{m}$ \\
\hline Diâmetro a $90 \%$ & $109,5 \mu \mathrm{m}$ & $72,83 \mu \mathrm{m}$ & $58,20 \mu \mathrm{m}$ & $43,17 \mu \mathrm{m}$ & $68,96 \mu \mathrm{m}$ \\
\hline
\end{tabular}

Tabela 2: Características físicas dos materiais.

\begin{tabular}{l|c|c|c}
\hline AMOSTRAS & $\begin{array}{c}\text { DIÂMETRO MÉDIO } \\
\text { DAS PARTÍCULAS } \\
(\boldsymbol{\mu m})\end{array}$ & $\begin{array}{c}\text { MASSA ESPECÍFICA } \\
\left(\mathbf{g} / \mathbf{c m}^{3}\right)\end{array}$ & $\begin{array}{c}\text { ÁREA SUPERFICIAL } \\
\left(\mathbf{m}^{2} \mathbf{g}\right)\end{array}$ \\
\hline CV NAT & 48,29 & 2,11 & 5,66 \\
\hline CV ULT UNI & 32,69 & 2,13 & 4,68 \\
\hline CV ULT SP & 22,32 & 2,25 & 5,66 \\
\hline CV SIF & 20,72 & 2,12 & 3,79 \\
\hline CV SIF+ULT UNI & 36,10 & 2,12 & 1,32 \\
\hline
\end{tabular}

Em relação aos valores de massa específica nas amostras analisadas, verificou-se que a CV ULT SP apresentou valor de massa específica maior comparado as demais amostras, com valor de $2,25 \mathrm{~g} / \mathrm{cm}^{3}$. Provavelmente este pequeno aumento pode estar associado ao método de tratamento sonoquímico que aplica as vibrações ultrassônicas através de uma haste de titânio, a qual notou-se um desgaste ao término do experimento. Diferente do equipamento Unique, onde a vibração foi realizada pela própria cuba, sem necessitar de haste. Segundo Adamian [19], durante um processo de cominuição, um problema muito comum que ocorre é a contaminação do material, principalmente quando se usa meios moedores de materiais metálicos. Salienta também que, embora se tenha verificado que a área superficial aumenta com o tempo de moagem, às vezes pode ocorrer uma aglomeração das partículas, produzindo o efeito contrário ao desejado.

Considerando os resultados apresentados de área superficial, não houve aumento da mesma em relação a amostra CV NAT comparada aos processos sonoquímicos (CV ULT UNI e CV ULT SP). Entretanto, quando comparado ao processo de sifonação (amostra CV SIF) onde buscou-se realizar uma prévia separação de fases (etapas 3 e 4 do processo de sifonação, conforme Figura 1), verificou-se o efeito contrário, ou seja, de $5,66 \mathrm{~m}^{2} / \mathrm{g}$ na CV NAT, diminui para $3,79 \mathrm{~m}^{2} / \mathrm{g}$. E quando aplicou-se o tratamento sonoquímico na amostra 
sifonada (CV SIF+ULT UNI), a área superficial diminui ainda mais, passando para 1,32 m²/g.

\subsection{Caracterização química e mineralógica}

O resultado analítico quantitativo que indica os elementos químicos inorgânicos presentes na forma de óxido mais estável realizado pela técnica de fluorescência de raios X (FRX) e o percentual de matéria orgânica (L.O.I) estão apresentados na Tabela 3. Verificou-se que a composição está de acordo com a literatura [3, 22] que apresenta um elevado percentual de sílica $\left(\mathrm{SiO}_{2}-60\right.$ a $\left.65 \%\right)$, alumina $\left(\mathrm{Al}_{2} \mathrm{O}_{3}-25\right.$ a 30\%) e magnetita $\left(\mathrm{Fe}_{2} \mathrm{O}_{3}-6\right.$ a $\left.15 \%\right)$.

Tabela 3: Composição química quantitativa da CV por FRX.

\begin{tabular}{l|l|l|l|l|l|l|l|l|l|l}
\hline AMOSTRA & \multicolumn{7}{|c}{ ÓXIDOS PRESENTES MAIS ESTÁVEIS (\%) } \\
\hline & $\mathrm{Al}_{2} \mathrm{O}_{3}$ & $\mathrm{CaO}$ & $\mathrm{Fe}_{2} \mathrm{O}_{3}$ & $\mathrm{~K}_{2} \mathrm{O}$ & $\mathrm{MgO}$ & $\mathrm{Na}_{2} \mathrm{O}$ & $\mathrm{SiO}_{2}$ & $\mathrm{SO}_{3}$ & $\mathrm{TiO}_{2}$ & L.O.I \\
\hline CV NAT & 26,43 & 1,23 & 3,42 & 1,50 & 1,19 & 1,77 & 59,34 & 0,27 & 1,08 & 3,77 \\
\hline
\end{tabular}

Analisando a composição semi-quantitativa realizada das fases cristalinas por difração de raios $\mathrm{X}$ (DRX) a CV NAT apresenta as fases de mulita $\left(3 \mathrm{Al}_{2} \mathrm{O}_{3} \cdot 2 \mathrm{SiO}_{2}\right)$ com $62 \%$, e quartzo $\left(\mathrm{SiO}_{2}\right)$ com $38 \%$, uma vez que estas fases são os principais constituintes cristalinos de cinza pobre em cálcio [3] e que são consideradas como cinzas de CLASSE C [10].

\subsection{Caracterização microestrutural}

A Figura 2 apresenta a morfologia observada para a amostra CV NAT, CV SIF como fração mais leve e menos densa e a CV residual como fração mais pesada e mais densa. Evidenciou-se que a CV NAT possui aglomerados de partículas e pleosferas de tamanhos que variam de 1 a $25 \mu \mathrm{m}$. Entretanto, na CV SIF concentrou-se mais partículas características da CV que apresentaram tamanhos mais homogêneos de média, aproximadamente $8 \mu \mathrm{m}$. A morfologia correspondente a CV Residual apresentou partículas maiores e/ou aglomerados de partículas de média, aproximadamente $27 \mu \mathrm{m}$.

A Figura 3 ilustra a CV após o tratamento sonoquímico. As imagens foram ampliadas 1500x e verificou-se uma melhor dispersão das esferas características da CV. Em ambos os equipamentos, os tamanhos analisados foram em média de aproximadamente $4 \mu \mathrm{m}$. A amostra CV ULT SP apresentou uma morfologia característica de pleosferas de tamanho maior com cenosferas em seu interior. Esta abertura da cavidade oca foi provavelmente ocasionada pelas ondas ultrassônicas [6,7]. Além disso, na amostra CV ULT UNI, percebeu-se um aglomerado com vários poros, possivelmente ocasionado também pelo efeito ultrassônico [6,7]. Este efeito pode ter acontecido durante os ciclos de compressão e expansão onde os gases e vapores são comprimidos para o interior da partícula e que durante a expansão são dirigidos para fora, com isso a cavidade aumenta gerando implosões no material [18].

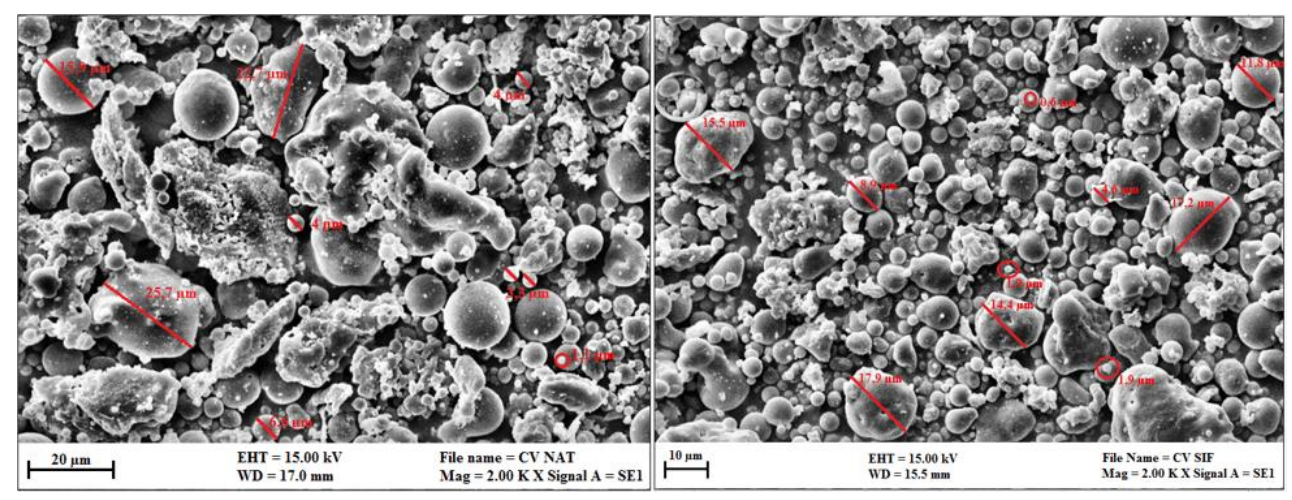




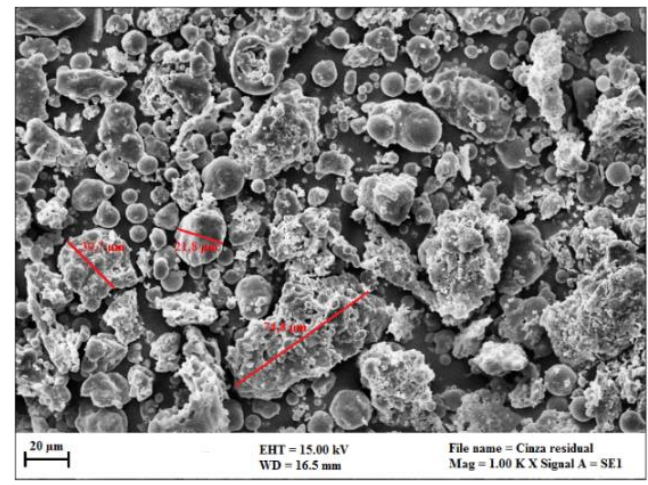

Figura 2: Imagens em MEV da CV NAT, SIF e Residual.

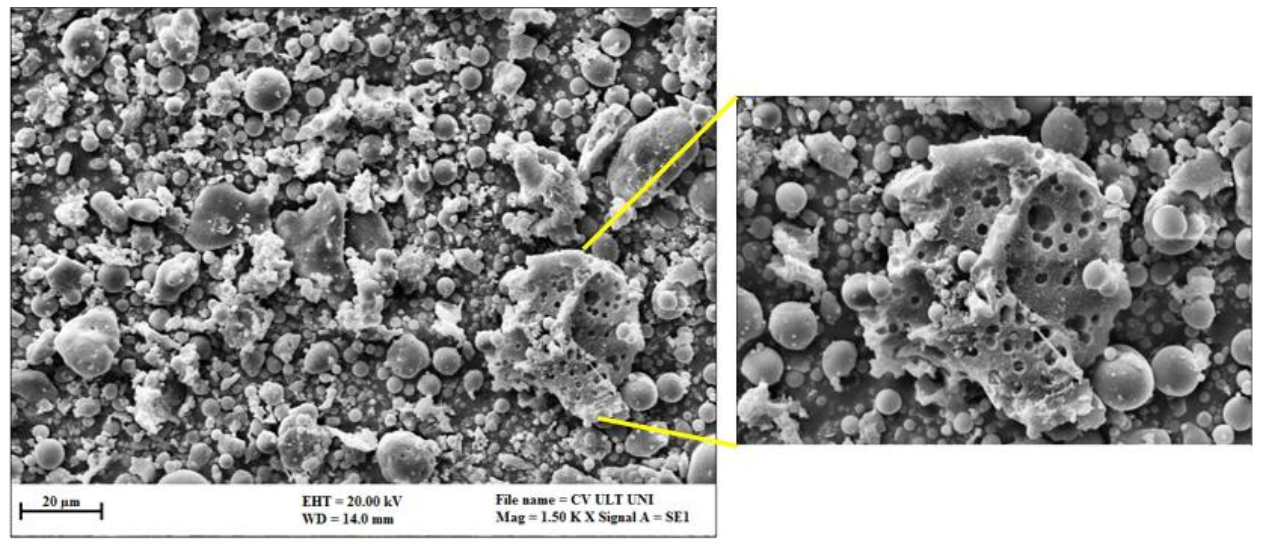

Figura 3: Imagens em MEV da CV ULT UNI.

\subsection{Avaliação do índice de atividade pozolânica}

Os resultados apresentados na Tabela 4 demostram que a amostra CV NAT não apresentava atividade pozolânica e após o tratamento sonoquímico passou a apresentar atividade pozolânica moderada nas amostras CV ULT SP e CV SIF, com valor mínimo para esta categoria. Entretanto, a combinação da sifonação mais o tratamento sonoquímico, na amostra CV SIF +ULT UNI, apresentou uma boa atividade pozolânica com valor de $1,3 \Delta \mathrm{mS} / \mathrm{cm}$, valor este acima do valor máximo do método que é correspondente à faixa de $\Delta \mathrm{mS} / \mathrm{cm} 0,8$ $<\mathrm{x}<1,2$. Neste caso, devido a uma maior diferença na medida da condutividade iônica da solução após a reação com $\mathrm{Ca}(\mathrm{OH})_{2}$, apresentou uma maior reatividade do material [13].

Os efeitos das adições minerais em cimentos são, usualmente, avaliados com base no consumo de portlandita, hidróxido de cálcio $\left[\mathrm{Ca}(\mathrm{OH})_{2}\right]$. A confirmação da atividade pozolânica depende também de uma série de características do material como: finura por peneiramento, massa e área específicas [12]. Portanto, além do ensaio de atividade pozolânica segundo método indireto por Luxàn et al. [13], realizou-se experimentalmente a moldagem de corpos de prova de acordo com a norma NBR 5752 [14]. De forma direta, avaliou-se o teor de cal fixado por atividade pozolânica que otimiza a interação da adição mineral com o hidróxido de cálcio, em virtude do procedimento da realização do ensaio, sob agitação $(16 \mathrm{~h} \pm 1 \mathrm{~h})$ e com temperatura controlada $\left(90^{\circ} \mathrm{C} \pm 5^{\circ} \mathrm{C}\right)[12]$.

A amostra CV ULT SP apresentou maior reatividade entre as demais amostras, com consumo de 633 mg Ca(OH) 2 /g de amostra, seguida da CV NAT, CV ULT UNI, CV SIF+ULT UNI e, por fim, a CV SIF. Os valores de consumo de cal são superiores ao consumo de $436 \mathrm{mg} \mathrm{Ca}(\mathrm{OH})_{2} / \mathrm{g}$ de amostra, valor mínimo estabelecido por Raverdy et al. [20] que classifica as adições minerais como material pozolânico.

Foram encontrados na literatura valores de consumo de $\mathrm{mg} \mathrm{Ca}(\mathrm{OH})_{2} / \mathrm{g}$ de amostra de cinza volante, sem qualquer beneficiamento ou tratamento, de $403 \mathrm{mg} \mathrm{Ca}(\mathrm{OH})_{2} / \mathrm{g}$ de amostra, em média [21]. O resultado encontrado na amostra CV SIF, indica um menor consumo de $\mathrm{Ca}(\mathrm{OH})_{2}$ em relação as demais amostras, apesar de ter apresentado um menor diâmetro médio de partícula. Segundo Ha et al. [22], a cinza volante que apresenta mais átomos de ferro na superfície das partículas tem maior reatividade em comparação àquelas com mais átomos de silício. Este fato explica, a sifonação realizada, onde a aproximação de partículas mais 
reativas com partículas de ferro, podem ter acelerado a sedimentação, fazendo com que permanecessem partículas suspensas (mais leves) e menos reativas, na maior parte, ligadas a partículas de sílica.

Tabela 4: Resultados de atividade pozolânica.

\begin{tabular}{|c|c|c|c|}
\hline \multirow{3}{*}{ AMOSTRAS } & \multicolumn{3}{|c|}{ ATIVIDADE POZOLÂNICA } \\
\hline & \multicolumn{2}{|c|}{ Método Luxàn } & \multirow{2}{*}{$\begin{array}{l}\text { Método Chapelle modificado } \\
\text { mg de } \mathrm{Ca}(\mathrm{OH})_{2} / \mathrm{g} \text { de amostra }\end{array}$} \\
\hline & $\Delta \mathbf{m S} / \mathbf{c m}$ & Resposta & \\
\hline CV NAT & 0,1 & Sem atividade pozolânica & 569 \\
\hline CV ULT UNI & 0,4 & Atividade moderada & 512 \\
\hline CV ULT SP & 0,4 & Atividade moderada & 633 \\
\hline CV SIF & 0,4 & Atividade moderada & 383 \\
\hline CV SIF+ULT UNI & 1,3 & Atividade pozolânica alta & 451 \\
\hline
\end{tabular}

Os resultados de resistência à compressão evidenciam que o método sonoquímico contribuiu para um aumento do índice de pozolanicidade do material conforme apresenta a Tabela 5. No tratamento combinado CV ULT UNI+SIF a resistência à compressão foi superior à referência em 112,9\%. O aumento da resistência aconteceu provavelmente, em sua maior parte, pelo efeito filler, facilitando os pontos de nucleação para os produtos de hidratação, e não apenas pela reatividade química, verificada pelo ensaio de Chapelle modificado. Segundo Dalmolin [5], o ensaio de resistência à compressão [14], talvez não seja adequado para avaliar o consumo de cal, pois o efeito filler, ou seja, o preenchimento dos espaços vazios, gera um aumento na densidade da mistura que altera a microestrutura da zona de transição, reduzindo a água retida sob os agregados que aumenta a resistência à compressão. A vantagem de avaliar a pozolanicidade por este método indireto, é que praticamente não há alteração na quantidade de água e não tem necessidade do uso de aditivos modificadores de viscosidade para ajustar a trabalhabilidade. Além disso, o consumo de cal é reduzido, deixando disponível $\mathrm{Ca}(\mathrm{OH})_{2}$ na mistura [23].

Tabela 5: Resultados de resistência à compressão para o ensaio de pozolanicidade da NBR 5752:2014.

\begin{tabular}{l|l|l|l|l}
\hline \multicolumn{1}{c|}{ AMOSTRAS } & \multicolumn{1}{|c|}{ REFERÊNCIA } & \multicolumn{1}{c|}{ CV NAT } & \multicolumn{1}{c}{ CV ULT UNI } & \multicolumn{1}{|c}{ CV ULT UNI+SIF } \\
\hline CP 1 & $28,01 \mathrm{MPa}$ & $24,45 \mathrm{MPa}$ & $29,54 \mathrm{MPa}$ & $32,2 \mathrm{MPa}$ \\
\hline CP 2 & $26,99 \mathrm{MPa}$ & $21,39 \mathrm{MPa}$ & $26,99 \mathrm{MPa}$ & $31,3 \mathrm{MPa}$ \\
\hline CP 3 & $25,46 \mathrm{MPa}$ & $21,39 \mathrm{MPa}$ & $28,52 \mathrm{MPa}$ & $29,0 \mathrm{MPa}$ \\
\hline CP 4 & $28,01 \mathrm{MPa}$ & $20,37 \mathrm{MPa}$ & $28,01 \mathrm{MPa}$ & $28,9 \mathrm{MPa}$ \\
\hline CP 5 & $25,46 \mathrm{MPa}$ & $24,96 \mathrm{MPa}$ & $30,56 \mathrm{MPa}$ & $30,2 \mathrm{MPa}$ \\
\hline CP 6 & $27,50 \mathrm{MPa}$ & $22,92 \mathrm{MPa}$ & $29,54 \mathrm{MPa}$ & $30,7 \mathrm{MPa}$ \\
\hline Média & $\mathbf{2 6 , 9 1} \mathrm{MPa}$ & $\mathbf{2 2 , 5 8} \mathrm{MPa}$ & $\mathbf{2 8 , 8 6} \mathbf{M P a}$ & $\mathbf{3 0 , 3 8} \mathrm{MPa}$ \\
\hline Desvio Padrão & $\mathbf{1 , 1 8}$ & $\mathbf{1 , 8 4}$ & $\mathbf{1 , 2 8}$ & $\mathbf{1 , 2 9}$ \\
\hline Índice de atividade pozolânica & - & $\mathbf{8 3 , 9 1 \%}$ & $\mathbf{1 0 7 , 2 5 \%}$ & $\mathbf{1 1 2 , 9 0 \%}$ \\
\hline
\end{tabular}

\section{CONCLUSÃO}

Quanto aos resultados de caracterização física, química, mineralógica e microestrutural realizados nos materiais, considerando os dados obtidos por análise de distribuição de tamanho de partículas [15], determinação de massa específica por picnometria a gás hélio, medida de área superficial pelo método BET, fluorescência de raios $\mathrm{X}$, difração de raios $\mathrm{X}$ e microscopia eletrônica de varredura, permitem as seguintes considerações:

- Na distribuição de tamanho de partículas, houve redução no diâmetro médio das partículas em comparação a CV NAT, considerando o tratamento ultrassônico aplicado. Na amostra CV ULT SP, encontrou-se um aumento de massa específica, uma vez que a mesma pode ter carregado contaminação do processo sonoquímico ocasionado próprio desgaste do equipamento com material titânio. O método BET utilizado para verificar a área superficial pode não ser o mais indicado para avaliar o aumento da reatividade física quando materiais são tratados com ondas ultrassônicas e sifonação. 
- A CV NAT apresentou valores de composição química semelhantes a valores encontrados na literatura [3, 22], bem como a composição semi-quantitativa mineralógica por difração de raios-X, composta basicamente pelas fases cristalinas mulita e quartzo, o que classifica o material como classe C, pobre em cálcio [3,10]. Neste momento, não foi possível avaliar a composição amorfa e cristalina da CV NAT.

- Na microestrutura por MEV verificou-se que o método sonoquímico tem energia para libertar as cenosferas das partículas de CV diminuindo consideravelmente o tamanho das partículas [9].

A utilização do método sonoquímico aplicado ao material mineral $\mathrm{CV}$, considerando os dados obtidos por meio do método indireto estabelecido por Luxàn et al. [13] e pela norma NBR 5752 [14], além do ensaio realizado por método direto estabelecido por Chapelle modificado, conforme Norma 15895 [12], permite as seguintes considerações:

- A determinação do índice de atividade pozolânica por Luxàn et al. [13], atribuiu pozolanicidade com atividade moderada nas amostras com tratamento sonoquímico, CV ULT UNI, CV ULT SP, CV ULT UNI+SIF, bem como na amostra CV SIF (atividade pozolânica alta) que sofreu apenas o processo de sifonação. Resultados comparados a CV NAT que não apresentou atividade pozolânica.

- Os resultados pertinentes ao ensaio Chapelle modificado indicam que todas as amostras analisadas apresentaram valores superiores a $436 \mathrm{mg} \mathrm{Ca}(\mathrm{OH})_{2} / \mathrm{g}$ de adição mineral, o que as qualifica como materiais característicos pozolânicos. As amostras CV NAT, CV ULT SP e CV ULT UNI são capazes de fixar maior quantidade de cal do a CV SIF. Entretanto, o índice de atividade pozolânica, por meio da hierarquização indireta (resistência à compressão) [14], indica um comportamento favorável com a possibilidade, inclusive, de desclassificá-la como material pozolânico, caso a CV seja utilizada na forma sifonada.

A amostra CV ULT SP não foi ensaiada quanto a NBR 5752 [14], pois a mesma foi enviada para o processo ultrassônico em outro estado, e a quantidade que foi autorizada para o ensaio não foi suficiente para a realização de todos os ensaios realizados.

\section{AGRADECIMENTOS}

Os autores agradecem ao LMC, LCVMat da Unisinos e à usina termelétrica de Candiota pela cinza volante disponibilizada para o estudo.

\section{BIBLIOGRAFIA}

[1] LEVANDOWSKI, J. E., KALKREUTH, W., "Chemical and petrographical characterization of feed coal, fly ash and bottom ash from the Figueira Power Plant, Paraná, Brazil”, International Journal of Coal Geology, v. 77, n. 3-4, pp. 269-281, Jan. 2009.

[2] ROCHA JUNIOR, C. A. F., SANTOS, S. C. A., SOUZA, C. A. G., et al. "Síntese de zeólitas a partir de cinza volante de caldeiras: caracterização física, química e mineralógica", Cerâmica, v. 58, n. 345, pp. 43-52, Ago. 2012.

[3] ALMARUZZAMAN, M., "A review on the utilization of fly ash", Progress in Energy and Combustion Science, v. 36, n. 3, pp. 327-363, Jan. 2010.

[4] HOPPE FILHO, J., GOBBI, A., PEREIRA, E., et al., “Atividade pozolânica de adições minerais para cimento Portland (Parte I): Índice de atividade pozolânica (IAP) com cal, difração de raios-X (DRX), termogravimetria (TG/DTG) e Chapelle modificado", Matéria, v. 22, n. 3, 2017.

[5] DALMOLIN, D. C. C., “Adições minerais”, In: $53^{\circ}$ Congresso Brasileiro do Concreto - Ibracon, São Paulo, 2011.

[6] SUSLICK, K. S. Kirk-Othmer Encyclodpedia of Chemical Technology, 4 ed., New York, John Willey \& Sons, 1998.

[7] GEDANKEN, A., "Using sonochemistry for the fabrication of nanomaterials", Ultrasonics Sonochemistry, v.11, n. 2, pp. 47-55, Abr. 2004.

[8] OHAYON, E., GEDANKEN, A., "The application of ultrasound radiation to the synthesis of nanocrystalline metal oxide in a non-aqueous solvent”, Ultrasonics Sonochemistry, v. 17, n. 1, pp. 173-178, Jan. 2010.

[9] LOURENÇO, R. R., EXPOSITO, C. C. D., ANGÉLICA, R. S., et al., "Ação sonoquímica e influência das condições de tratamento térmico na preparação de cimentos do sistema binário $\mathrm{CaO}-\mathrm{Al}_{2} \mathrm{O}_{3}$ ”, Cerâmica, v. 56, pp. 28-38, Mar 2010.

[10] METHA, P.K., MONTEIRO, P.J.M. "Concreto: Microestrutural, Propriedades e Materiais”, 2 ed., chapter 8, São Paulo, Brasil, Ibracon, 2008. 
[11] ASSOCIACAO BRASILEIRA DE NORMAS TECNICAS. NBR 12653: Materiais Pozolânicos, Rio de Janeiro, ABNT, 1992.

[12] ASSOCIACAO BRASILEIRA DE NORMAS TECNICAS. NBR 15895: Materiais pozolânicos - Determinação do teor de hidróxido de cálcio fixado - Método Chapelle modificado, Rio de Janeiro, ABNT, 2010.

[13] LUXÀN, M. P., MADRUGA, F., SAAVEIRA, J., et al. "Rapid evaluation of pozzolanic activity of natural products by conductivity measurement", Cement and Concrete Research, v. 19, n. 1, pp. 63-68, Jan. 1989.

[14] ASSOCIACAO BRASILEIRA DE NORMAS TECNICAS. NBR 5752: Materiais pozolânicos - determinação do índice de desempenho com cimento Portland aos 28 dias, Rio de Janeiro, ABNT, 2014.

[15] INTERNATIONAL ORGANIZATION FOR STANDARDIZATION. ISO 13320: particle size analysis - Laser diffraction methods (E) content. Geneva, ISO, 2009.

[16] ASSOCIAÇÃO BRASILEIRA DE FUNDIÇÃO. CEMP 120: Determinação da perda ao fogo, São Paulo, ABIFA, 2003.

[17] ASSOCIACAO BRASILEIRA DE NORMAS TECNICAS. NBR 7215: Cimento Portland - Determinação da resistência à compressão, Rio de Janeiro, ABNT, 1997.

[18] MARTINES, M. A. U., DAVOLOS, M. R., JÚNIOR, M. J., et al. "O efeito do ultra-som em reações químicas", Química Nova, v. 23, n. 2, pp. 251-256, Jan. 2000.

[19] ADAMIAN, R. "Novos Materiais: Tecnologia e Aspectos Econômicos", 1 ed., chapter 5, Rio de Janeiro, Brasil, COPPE, UFRJ, 2009.

[20] RAVERDY M., BRIVOT F., PAILLERE A.M., et al., "Appreciation de I'activite pouzzolanique des constituants secondaires", In: 7th Int. Congr. Chem. Cem. Paris, 1980.

[21] QUARCIONI, V. A., CHOTOLI, F. F., COELHO; A. C. V., et al., "Indirect and direct Chapelle's methods for the determination of lime consumption in pozzolanic materials", Revista Ibracon de Estruturas e Materiais, v. 8, n. 1, pp. 1-7, February 2015.

[22] HA, J., CHAE, S., CHOU, K. W., et al., "Characterization of class F fly ash using STXM: identifying intraparticle hererogeneity at nanometer scale", Journal of Nanomaterials, 2016.

[23] HOPPE FILHO, J., GOBBI, A., PEREIRA, E., et al., "Atividade pozolânica de adições minerais para cimento Portland (Parte II): Índice de atividade pozolânica com cimento Portland (IAP), difração de raios-X (DRX) e termogravimetria (TG/DTG)", Matéria, v. 22, n. 3, 2017. 